\title{
小学数学教学中如何培养学生的解题能力
}

齐玉伟

黑龙省绥芬河市北寒小学

DOI:10.32629/jief.v2i5.1467

[摘 要] 小学数学是基础数字和加减法的认识阶段, 高年级开始接触应用题解题, 能够帮助小学生逻辑思维发展。数学作为帮助学生拓展思 维的学科, 在小学教学中, 老师要不断融入创新教学方式, 注重引导学生的思维发展, 从小建立和培养学生的解题能力, 让学生能够对数学 产生兴趣, 保持对未解的数学题目的探索精神, 促进思维发展, 更好地掌握数学规律。

[关键词] 小学数学; 解题能力; 培养

中图分类号: G633.6 文献标识码: A

对教学课标的应用, 突破了传统教育的教学方式, 不再是老师对理 论知识进行喋喋不休的 “传道受业解惑”, 而是在教学的过程中, 学生也 要积极、主动的参与到其中与老师进行互动, 在这样的过程中, 老师要 做的是引导学生应用正确的数学思考方式, 应用正确逻辑思维方式去学 习数学, 且要具有因材施教教学方式。学生主动的参与到数学学习与探 究的过程中, 这样有助于学生对数学解题能力的培养。

\section{1 培养小学生数学解题能力的重要性}

1.1 促进小学生综合全面发展

而目前的教育行业, 由于不重视小学生的思维发展, 很多老师还是 采用灌输式教学, 没有激起学生探究数学规律的积极性, 学生在认识数 学的时候只感觉到枯燥乏味, 对数学感到吃力, 数学成绩很难提高, 解 题能力也无法得到有效提升。现代教育逐渐转向培养学生综合素质发展, 在小学生的基础知识教学阶段, 老师不能再以分数划分学生好坏, 而是 要着重关注小学生思维逻辑发育, 数学本身就是属于逻辑性很强的学科, 小学生除了认识数字和掌握基本计算能力之外, 还能促进思维逻辑能力 的培养。

\section{2 学会发现数学规律, 掌握技巧}

小学生比较活泼好动, 在进行数学教学的时候, 如果老师没有充分 激发学生的学习兴趣, 孩子很难理解消化枯燥的数学理论知识, 进行数 学问题解答的时候, 也不会灵活转变思维, 运用解题技巧, 更加不会主 动提问老师解答疑问。小学阶段的孩子, 思维是最活跃的时期, 对任何 新鲜事物的学习都能够快速掌握。在进行数学教学时, 除了基本的课本 知识教学, 掌握基础知识之外, 老师要着重关注学生的思维发育, 引导 学生对数学保持兴趣, 学会深入探索问题, 发现数学隐藏的规律, 掌握 解题技巧, 提升解题能力。

\section{2 培养小学生数学解题能力的策略}

2.1 激发学生学习的兴趣

新课标的产生, 与过去的教学方式大相径庭, 学生与老师之间互动 增加, 使学生在学习数学的过程中, 更加有突破性的思维方式。改变了 将老师认为是重点的地方, 对学生进行讲解, 学生通过被动接受的方式, 然后紧接着通过对练习题来获取数学知识。通过这样的一种方式教学, 学生是没有办法对数学知识有全面的了解, 同时会让学生感到枯燥无味, 无法对学到的知识进行举一反三的学习。而新课标的引入, 可以将生活 中的实际例子引入到其中, 如学生在对长方形、正方形求面积与周长是, 可以将学生身边的事物采取利用的方式, 如书桌、乒乓球台等, 以实际 事物来对题目进行讲解, 通过生活中的实践加入到教学的过程中, 培养 学生的实践探究能力, 与学习数学的兴趣, 理论与实践相结合, 使数学
学习更加的有趣。

2.2 提高学生的积极参与意识与培养问题意识

新课标, 学生可以充分的发挥自己天马行空的想象, 在课堂上与教 师进行探讨, 摆脱掉了以往教师固定传授知识的模式。这样的教学方式 对学生培养提出问题与探究有一定的帮助。因为学生在学习的过程中, 通过与教师的相互探讨, 然后探究出适合自己的学习数学方式; 新课标 的引入, 所实行的主要目的是要让学生积极主动的参与到学习数学的兴 趣中, 然后通过与老师互动, 启发学习数学的逻辑思维方式是否正确, 同时也是自我修养学习数学的一种行为意识。教师的主要宗旨就是, 将 教学大纲进行一定的疏通, 让学生自主的学习数学, 然后具有针对性的 去启发学生独立思考的能力与正确的方法, 对数学问题的解决方式不断 的进行挑战, 追求知识的欲望不断的提高, 学生积极的参与学习, 对知 识进行全面的掌握。

\section{3 以小学生思维发展为主, 制定合适的教学方案}

小学生数学解题能力的培养, 关键在于教师的教学方法是否符合小 学生思维发展。老师作为成年人, 进行教学的时候肯定是以自己的思维 方式为主, 但是小学生还处于思维发育期, 很多在老师看来简单的知识, 小学生需要花费大量的时间和精力去消化。而且小学生处于活泼好动的 阶段, 容易被其他事物吸引, 上课的时候很难集中注意力, 老师教学时 就要根据孩子的状态, 制定好教学方案, 成功吸引孩子的注意力, 让孩 子能够对数学知识产生兴趣, 这样才能够集中精神听课, 理解数学知识, 在老师进行提问的时候, 也能够积极主动地举手回答问题, 遇到不懂的 地方会主动询问求知, 老师再加以适当的引导, 促进学生思维发展, 数 学解题能力自热就能得到提升。

\section{3 结语}

数学知识涵盖范围广阔, 逻辑性强, 老师要不断提升优化自身教学 水平, 跟着时代发展创新教学方式, 利用多媒体开展情境化教学。在教 学过程中, 老师要注意小学生的天性发展, 促进学生思维逻辑发展, 让 学生的解题能力得到有效提高, 面向综合全面发展。

\section{[参考文献]}

[1]胡虎林, 马雅娉. 小学数学教学中培养学生问题意识及能力之实践 研究[J].学周刊,2019(33):39.

[2]刘艳霞. 浅析小学高年级数学读题解题能力的培养[J]. 课程教育 研究,2019(37):181.

[3]陈再明. 有效构建小学数学互动课堂策略 [N]. 贵州民族 报,2020-08-27(B03). 